\title{
Studies of the Agarose Gelling System by the Fluorescence Polarization Method. I.*
}

\author{
Akira Hayashi, Kyosuke Kinoshita, and Masaru Kuwano \\ Department of Chemistry, Ritsumeikan University, Kyoto 603, Japan.
}

(Received November 19, 1976)

\begin{abstract}
The agarose gelling system was investigated with the fluorescence polarization method using fluorescent dyes, such as uranine (fluorescein sodium), as an extrinsic probe. The method detected three critical points, $c_{1}, c_{2}$, and $c_{3}$, in the concentration change of agarose at room temperatare, corresponding to the concentrations at which the agarose domain occupies the whole solution, the junction zone is made to gel, and the network is presumably completed to hold gel elasticity, respectively. Three transition points, $T_{1}, T_{2}$, and $T_{3}$, were also detected in the temperature range studied for these systems. $T_{1}$ and $T_{3}$ are well defined transition points of agarose, the gel point and the melting point. $\mathrm{T}_{2}$ is an unknown, new transition point. In order to examine the transition at $\mathrm{T}_{2}$, some other solution properties were determined with the fluorescence polarization method, and $T_{2}$ was interpreted to be the coil-helix transition temperature of agarose. Thus the fluorescence polarization method provides useful information for the investigation of a polymer gelling system such as agarose.

KEY WORDS Fluorescence Polarization Method / Agarose / Sol-

Gel Transition / Coil-Helix Transition / Hysteresis /
\end{abstract}

Fluorescence methods have been applied to estimate the inner, microscopic structure of various polymer systems including the solid, melt, and solution states. ${ }^{1}$ The methods consist of an evaluation of the emission characteristics of fluorescence from extrinsic or intrinsic probes involved in the polymer system.

In the present work, the fluorescence polarization method was to be applied for the gelling system of natural polymers such as agarose. The principle of the method is based upon Perrin's equation:

$$
\begin{aligned}
\left(\frac{1}{p}-\frac{1}{3}\right) & =\left(\frac{1}{p_{0}}-\frac{1}{3}\right)\left(1+\frac{3 \tau}{\rho}\right) \\
& =\left(\frac{1}{p_{0}}-\frac{1}{3}\right)\left(1+\frac{k T}{v \eta} \tau\right)
\end{aligned}
$$

where $\tau, \rho, v$, and $p_{0}$ are the lifetime, the rotational relaxation time, the volume of the

* Presented at the 25th Annual Meeting of the Society of Polymer Science, Tokyo, Japan: Polymer Preprints, Japan, 25 (2), 250 (1976). fluorescence probe, and the limiting value of the degree of polarization for $\rho \rightarrow \infty$, respectively, and $\eta$ is the local viscosity. The degree of polarization of fluorescence, $p$, is thus related to the rotational relaxation time or the local viscosity through the equation. If $\tau$ is measured for the system, this condition is not necessary. These values, $\rho$ and $\eta$, are not the direct measure of the polymer motion itself but that of the fluorescence probe added to the polymer system. The motion of a probe molecule, however, reflects the microscopic environment and therefore information on the internal structure of the polymer system can be obtained.

In a reversible polymer gel such as agar, it is known that non-covalent bondings from junction zones among polymer chains to cause gelation, and the inner, microscopic properties such as electric conductivity change only slightly even though the macroscopic appearance of the solution changes drastically on gelation.

The gelling polymer used here was agarose, 


\section{A. Hayashi, K. Kinoshita, and M. Kuwano}

a gelling fraction of agar polysaccharide; its chemical structure was established by Araki. ${ }^{2}$ Agarose is a naturally occurring biopolymer which has a stereoregular repeating unit and is known to have a more complex transition behavior, such as a coil-helix transition and hysteresis phenomena, than in the case of synthetic polymer gels. The gelling mechanism of agarose has also been investigated by many researchers. ${ }^{3}$ Among them, Rees, et al., ${ }^{4}$ especially have carried out active work relating to carrageenan, ${ }^{5}$ another typical gelling polysaccharide obtained from seaweed.

In the present paper, the fluorescence polarization method is effectively applied to follow the agarose sol-gel transition process, and the transition points detected by the method are related to those characterized by other methods.

\section{MATERIALS AND EXPERIMENTS}

\section{Materials}

The sample of agarose was "Agarose A-37" (Nakarai Chemicals Ltd., Kyoto, Japan), which contained $0.30 \%$ of sulfate and $3.03 \%$ of D-glucuronic acid with none of the pyruvic acid residues. The fluorescence dyes used as probes were uranine, thionine, and Whitex RP (Sumitomo Kagaku Ltd.).

\section{Fluorescence Polarization Method}

The instrument used was a Hitachi Fluorescence Spectrophotometer 204 with polarizer and analyzer. The optical axis of the polarizer was fixed vertically. The degree of polarization was calculated with the following equation: $p=$ $\left(I_{\| /}-I_{\perp}\right) /\left(I_{\| /}+I_{\perp}\right)$, where $I_{\| /}$and $I_{\perp}$ are the polarized components of fluorescence intensity for parallel and perpendicular alignments of polarizer and analyzer, respectively. Agarose was dissolved at $85^{\circ} \mathrm{C}$ and then uranine solution was added. The cell containing the sample solution was placed in the cell holder which had a jacket for circulating the temperaturecontrolled water. The combinations of wavelengths of exciter and analyzer beams for measurement of the degree of polarization were $465 \mathrm{~nm}$ and $525 \mathrm{~nm}, 570 \mathrm{~nm}$ and $630 \mathrm{~nm}$, and $380 \mathrm{~nm}$ and $450 \mathrm{~nm}$, for uranine, thionine, and Whitex RP, respectively.

\section{Other Measurements}

Optical rotation was measured with a Hitachi Spectrophotometer EPU with an optical rotation attachment; the wavelength and pass-length of the cell were $572 \mathrm{~nm}$ and $10 \mathrm{~cm}$, respectively. The differential scanning calorimeter (DSC) thermogram was measured with a Perkin-Elmer DSC 1B with sealed pan. An Ubbelohde-type viscometer was used for the measurement of reduced viscosity.

\section{RESULTS}

\section{Changes in Degree of Polarization with Agarose} Concentration

The polarization, $p$, was determined at $25^{\circ} \mathrm{C}$ for the solutions or gels containing $0-0.3 \mathrm{~g} / \mathrm{d} l$ of agarose, with $10^{-6} M$ of uranine as an extrinsic probe. Reciprocals of the degree of polarization are plotted against agarose concentration in Figure 1, in which the plots measured with thionine or Whitex RP are also included. All these curves show abrupt changes at 0.12 and $0.24 \mathrm{~g} / \mathrm{d} l$ of agarose concentration, which are designated as $c_{1}$ and $c_{2}$.

The $c_{2}$ agrees with the apparent gelling concentration at which the solution loses its fluidity, but $c_{1}$ was not observable from the solution appearance. In order to reexamine $c_{1}$ and $c_{2}$, the degree of polarization and the reduced viscosity of the agarose solution were determined at $50^{\circ} \mathrm{C}$, at which no gelation occurs. The results are shown in Figure 2. A polarization change at $c_{1}$ still remains, corresponding to that at $25^{\circ} \mathrm{C}$, but the one at $c_{2}$ disappears because of the non-gelling temperature. The reduced viscosity does not show any marked changes corresponding to those of polarization but rises gradually below $c_{1}$, showing a weak polyelectrolytic behavior. Thus $c_{1}$, which is the first abrupt change of polarization in the low polymer concentration range, seems to be the concentration at which the expanded agarose domain occupies the whole volume of the solution. ${ }^{12}$

Agarose gel became turbid at concentrated solutions above $0.3 \mathrm{~g} / \mathrm{d} l$ and the accurate determination of polarization became difficult. In order to avoid this disadvantage, our measurement was carried out using 36-\% sugar solution as a solvent instead of distilled water. 
Studies of Agarose Gel by Fluorescence Method. I.

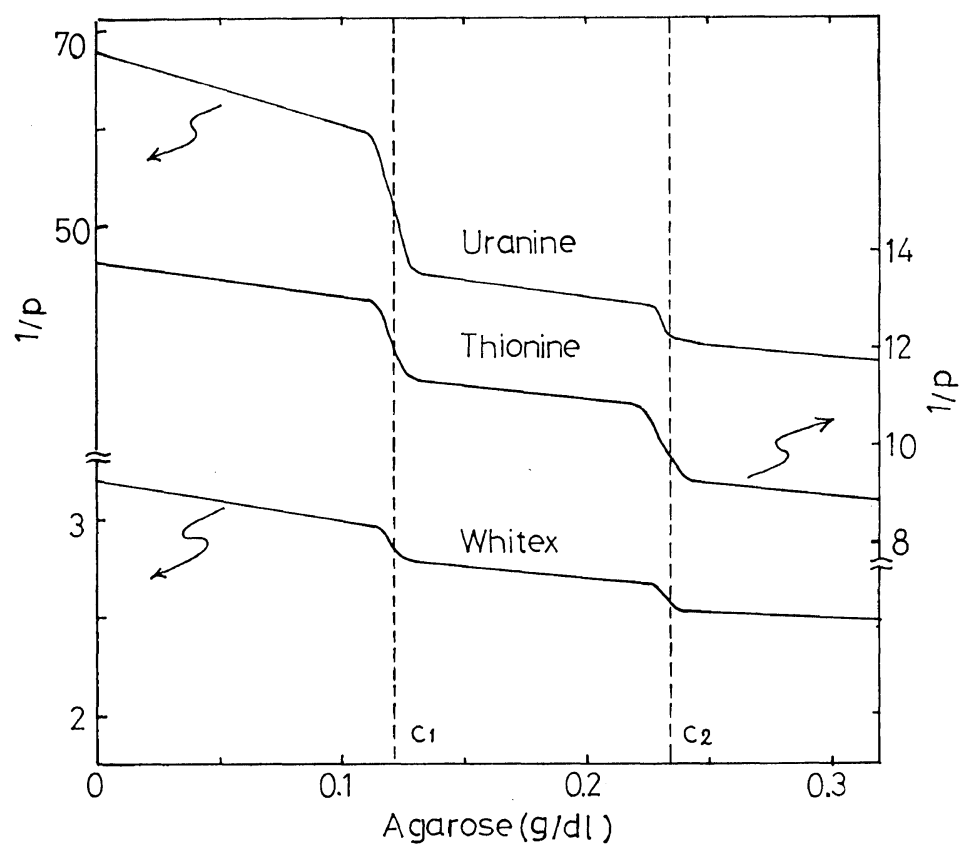

Figure 1. Changes in reciprocal of degree of polarization $(1 / p)$ with agarose concentration at $25^{\circ} \mathrm{C}$.

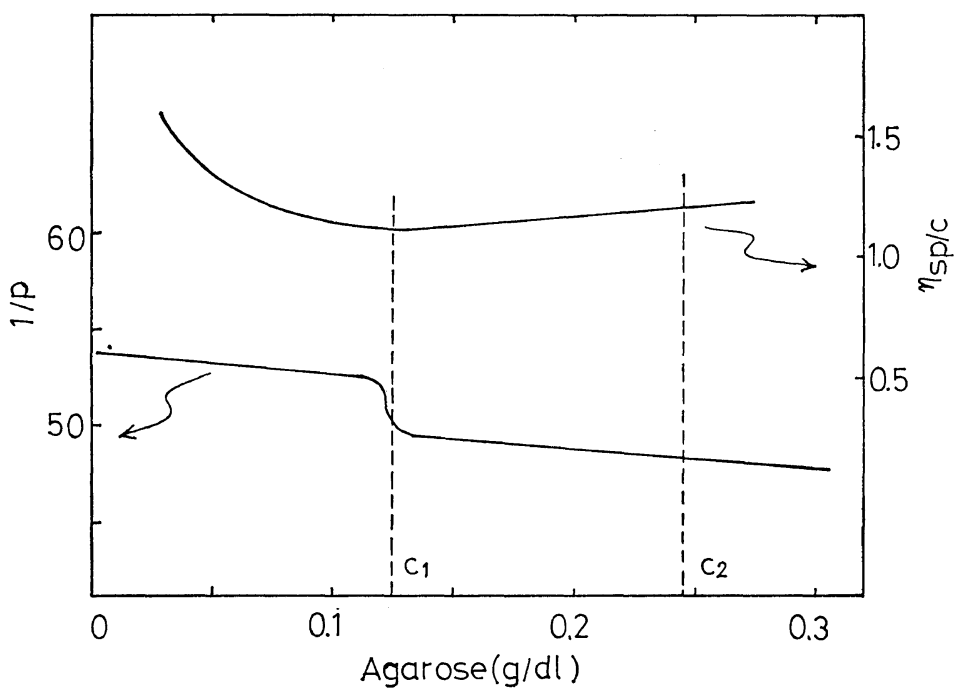

Figure 2. Changes in $1 / p$ and reduced viscosity with agarose concentration at $50^{\circ} \mathrm{C}$.

Thus measurements became possible with the sugar solution up to $1.5 \mathrm{~g} / \mathrm{d} l$ of agarose, as shown in Figure 3. Another change in addition to the two changes at $c_{1}$ and $c_{2}$ is observed at about $1.0 \mathrm{~g} / \mathrm{d} l$ of agarose, this is designated as $c_{3}$.
Changes in the Degree of Polarization and Other Solution Properties with Temperature

Preliminary tests in the warming test tube showed that agarose A-37 gelled at about $40^{\circ} \mathrm{C}$ and its gel melted at about $78^{\circ} \mathrm{C}$. Polarization changes of the agarose solution were determined 


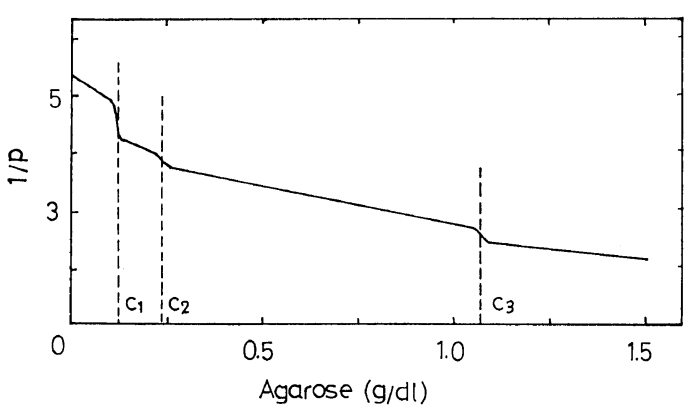

Figure 3. Changes in $1 / p$ of agarose dissolved in $36-\%$ sugar solution with agarose concentration at $25^{\circ} \mathrm{C}$.
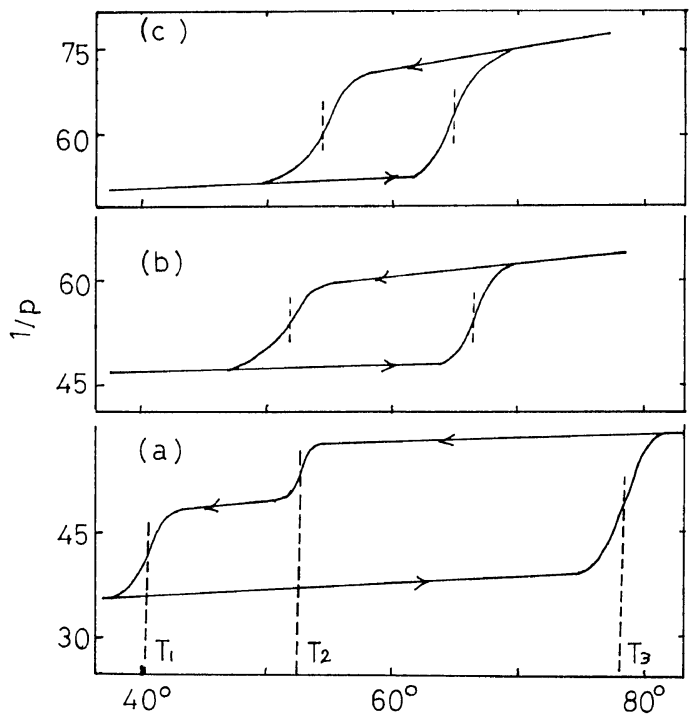

Figure 4. Changes in $1 / p$ of (a) $0.3-\mathrm{g} / \mathrm{d} l$, (b) $0.15-\mathrm{g} / \mathrm{d} l$, and (c) $0.05-\mathrm{g} / \mathrm{d} l$ agarose solution with temperature.

under decreasing temperature from $85^{\circ} \mathrm{C}$ to $30^{\circ} \mathrm{C}$ and in reverse from $30^{\circ} \mathrm{C}$ to $85^{\circ} \mathrm{C}$. Temperature intervals of the measurements were $1^{\circ}$ or $3^{\circ}$, and the temperature was maintained constant for at least $10 \mathrm{~min}$ before the measurement. The extension of the maintenance time from $10 \mathrm{~min}$ to $30 \mathrm{~min}$ did not cause any distinguishable differences in the degree of polarization determined.

Figure $4 \mathrm{a}-\mathrm{c}$ show the typical results of $0.3 \mathrm{~g} / \mathrm{d} l\left(>c_{2}\right), 0.15 \mathrm{~g} / \mathrm{d} l\left(<c_{2},>c_{1}\right)$, and $0.05 \mathrm{~g} / \mathrm{d} l$ $\left(<c_{1}\right)$ of agarose, respectively. These curves show apparent hysteresis loops even in the cases

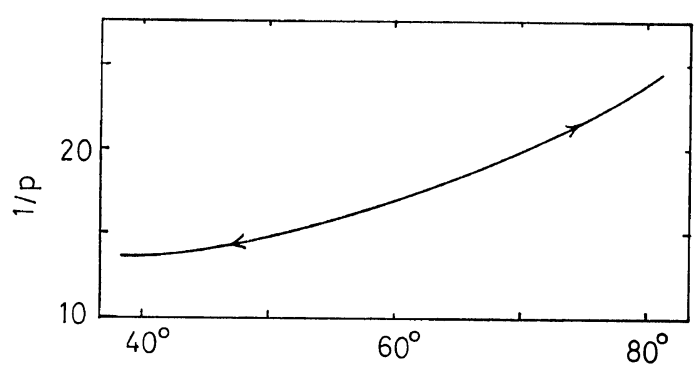

Figure 5. Changes in $1 / p$ of $0.3-\mathrm{g} / \mathrm{d} l$ agarose dissolved in $2 N$ potassium thiocyanate solution with temperature.

of nongelling solutions, 0.15 and $0.05 \mathrm{~g} / \mathrm{d} l$ : the pathway of decreasing temperature differs from that for increasing temperature within a certain temperature range. In the gelling solution, $0.3 \mathrm{~g} / \mathrm{d} l$, two distinct changes are observed at about $40^{\circ} \mathrm{C}$ and $53^{\circ} \mathrm{C}$ in decreasing temperature, and the third change at about $78^{\circ} \mathrm{C}$ in the increasing. They are designated as $T_{1}, T_{2}$, and $T_{3}$, respectively. These transition temperatures were slightly shifted to higher temperatures upon an increase in agarose concentration. $\mathrm{T}_{1}$ and $T_{3}$ correspond to the gel point (or setting point) and the melting point, but $T_{2}$ is an unknown transition point with no apparent structural change. In the curves in Figures $4 \mathrm{~b}$ and $\mathrm{c}, \mathrm{T}_{1}$ is not observed because of the nongelling concentration, but the unknown change at $\mathrm{T}_{2}$ still remains. Another change in increasing temperature in Figures $4 \mathrm{~b}$ and $\mathrm{c}$ seems to correspond to the melting point, $T_{3}$, even through the solution does not gel macroscopically.

In order to reexamine these transitions, especially that at $T_{2}$, the degree of polarization was determined for the agarose solution in $2 \mathrm{~N}$ potassium thiocyanate, which disturbs hydrogen bonding among agarose molecules to cause no gel. A typical example of $0.3 \mathrm{~g} / \mathrm{d} l$ solution of agarose is shown in Figure 5. The curve has no abrupt changes either in increasing temperature or decreasing, and is completely reversible in both pathways. This result means that hydrogen bonding causes the transition at $T_{2}$ as well as the gelation at $T_{1}$.

Some solution properties were determined around $T_{2}$. Among them, the results of optical rotation, reduced viscosity, and differential scan- 
Studies of Agarose Gel by Fluorescence Method. I.

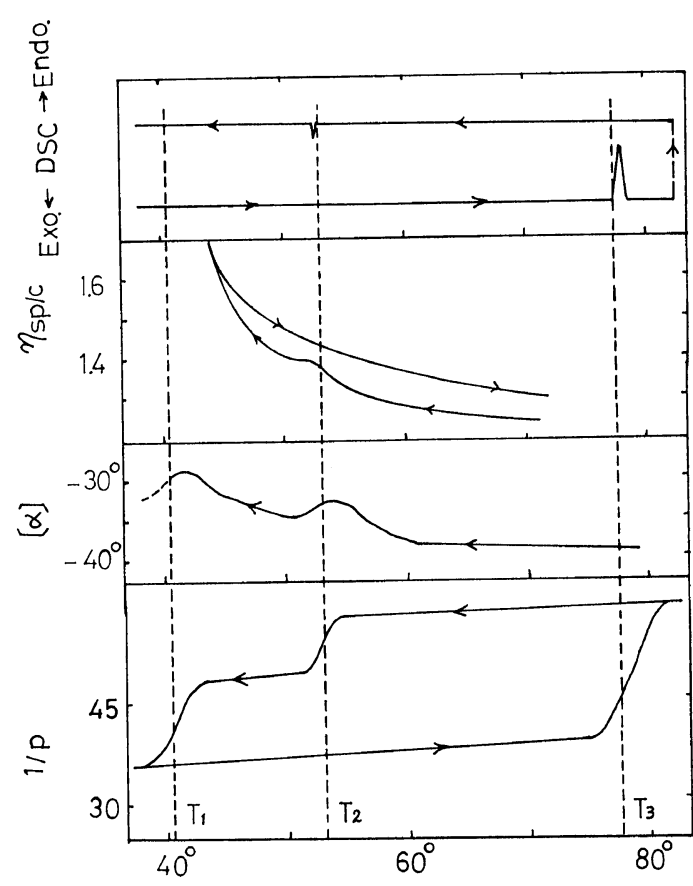

Figure 6. Changes in optical rotation, reduced viscosity, and DSC thermogram of $0.3-\mathrm{g} / \mathrm{d} l$ agarose solution with temperature.

ning calorimetry gave some interesting correspondences to that of fluorescence polarization, as shown in Figure 6. Electric conductivity and refractive index showed apparent, but very slight, changes at $T_{2}$ only in the course of decreasing temperature, showing certain corresponding changes in the agarose solution, but the results are not cited here because the change is too small and the interpretation is difficult. In Figure 6, typical results from the three methods on $0.3-\mathrm{g} / \mathrm{d} l$ solution of agarose are compared with that of the fluorescence method. The optical rotation shows two peaks near $T_{2}$ and $\mathrm{T}_{1}$, which suggests some conformational changes of the agarose molecule in the system. The reverse curve on increasing temperature could not be determined because of the turbidity of the gel and of the insufficient sensitivity of the apparatus used.

The reduced viscosity changes irregularly at $\mathrm{T}_{2}$ on decreasing temperature but not on increasing temperature. The curve shows a hysteresis corresponding to that observed by the fluores- cence method. The irregular change of reduced viscosity suggests a change of hydrodynamic form of agarose chains at $T_{2}$. The most interesting peaks are observed in the DSC curve: a smaller exothermic peak at $T_{2}$ in decreasing temperature and another bigger endothermic one at $T_{3}$ in increasing. There are no peaks at the gel point, $T_{1}$, either in decreasing or increasing. The peak in the DSC curve means a transition accompanying the endothermic or exothermic reaction. The transition at $T_{2}$ seems therefore to be an exothermic irreversible reaction observed only in the temperature decreasing pathway; such a reaction has never been reported. The meaning of this transition point will be discussed in the following section.

\section{DISCUSSION}

It has been shown by Duckworth and Yaphe that agar is a complex mixture of polysaccharides, all having the same backbone structure but substituted to a variable degree with charged groups. Neutral agarose composed of only alternating (1-4)-3,6-anhydro-L-galactosyl-(1-3)-Dgalactose residues is, therefore, an oversimplification and is an extreme of structure in the spectrum of polysaccharides. Agarose A-37 used here is not free from this question and actually behaved as a weak polyelectrolyte, as shown in Figure 2.

In Figure 1, $c_{1}$ corresponds to the concentration at which the whole solution is just filled with agarose domains expanded by the electrostatic repulsions. The critical concentration, $0.12 \mathrm{~g} / \mathrm{d} l$, is much smaller than that of other aqueous polymer solutions, for example, 0.5$2.0 \mathrm{~g} / \mathrm{d} l$ for polyacrylamide solution. ${ }^{1 \mathrm{a}}$ The low value is owing to the rigid polysaccharide structure and the polyelectrolytic expansion of the agarose chain. The gelling concentration, $c_{2}$, is also lower than those of the usual gels, probably for the same reason. At this concentration, an overlapping of agarose domains allows the formation of enough intermolecular junction zones to make a gel. The meaning of the third critical point at $c_{3}$ near $1.0 \mathrm{~g} / \mathrm{d} l$ in sugar solution in Figure 3 has not been examined further, but seems to correspond to the changing point of gel elasticity reported 
by Hirai, ${ }^{7}$ who suggested that the network of agarose was completed at this concentration.

Three different fluorescence probes, uranine, thionine, and Whitex RP, all show the same $c_{1}$ or $c_{2}$ in the $1 / p-c$ curves in Figure 1 , but the $1 / p$ values of each are quite different at the same agarose concentration. The degree of polarization, $p$, is a function of $\rho$ and $\tau$, and then $\rho$ is a function of $v$ and $\eta$ as shown by Perrin's equation. Therefore, the difference of $1 / p$ among the three probes seems to be caused mainly by those of $\tau$ and $v$ of the probes under the experimental conditions. The abrupt change of $1 / p-c$ curve of a certain probe at $c_{1}$ or $c_{2}$ (also at $c_{3}$ ) reflects the change in $\rho$ of the probe molecule itself, but the corresponding change of $v$ or $\eta$ is not expected in such a diluted agarose solution as used here. Tne change in $\tau$ will not be discussed here because the fluorescence intensity $\left(=I_{\| /}+2 I_{\perp}\right)$ did not change so abruptly in the course of the determinations.

A fluorescence probe such as uranine seems to be adsorbed on agarose chains in the system and the $\rho$ of the adsorbed probe should be controlled by the motion of the agarose chains. If the motion or relaxation time of the agarose chain changed suddently at $c_{1}$ or $c_{2}$, at which an overlap or network of the chain segment occurs, $\rho$ of the adsorbed probe and hence $1 / p$ of the system should be correspondingly changed. ${ }^{*}$ This interpretation is also useful in the following discussion about $T_{1}, T_{2}$, and $T_{3}$.

The most interesting feature of the phase transition in agarose system is undoubtedly that

\footnotetext{
* Some difficulty is expected in examining the adsorption of the probes, such as uranine, on polymer chains in the diluted solution, or gel systems. Fortunately, it has been found that the diluted agarose gel is broken and precipitated by the freeze-thaw process of the gel (D. A. Rees, et al., J. Mol. Biol., 68, 158 (1972)). The phenomenon was employed in the agarose-uranine gel system for checking the uranine adsorption on agarose chains. As the result, it was found that uranine was positively adsorbed on agarose chains according to the Freundlich equation. It is quite interesting that the adsorption of probes on polymer chains takes part in the detection of the transition points by the fluorescence polarization method. The detailed data will be reported in a subsequent paper of this series.
}

it changes with temperature. Figure 4 shows the typical hysteresis loops including three transition points, $T_{1}, T_{2}$, and $T_{3}$, and also shows that the fluorescence polarization method is applicable with success to the investigation of the agarose gelling system.

The meanings of $T_{1}$ and $T_{3}$ are apparent: that is, they are the gel point and the melting point, respectively. $T_{2}$ is the unknown, new transition point. At $T_{2}$ in the temperature decreasing pathway, optical rotation, reduced viscosity, and DSC curves in Figure 6, and electric conductivity and refractive index data not cited here, all show apparent changes, as well as in the case of fluorescence polarization.

Pines and Prins ${ }^{8}$ and Rees, et al., ${ }^{4}$ estimated agarose hysteresis loops by the optical rotation method, but overlooked the $\mathrm{T}_{2}$ transition point. This might be because of the smaller change at $T_{2}$ than that at $T_{1}$ in using the optical rotation method. We purchased agarose powder (batch code: REX 5468) from Marine Colloids Inc., Rockland, Maine, U.S.A., which was used in Rees' work, and found that it also had a $\mathrm{T}_{2}$ transition at the same temperature as agarose A-37. Therefore, the $T_{2}$ transition is not a special feature of agarose A-37, but a common one in agarose. In their works on carrageenan gel, Rees, et al., ${ }^{9}$ found two critical points in their optical rotation curves, and proposed them to be the coil-helix transition point and the gel point, respectively. Then, they suggested in a subsequent paper, that the optical rotation change at the coil-helix transition is caused by the torsion of bond angles around the glycosidic oxygen bridges in carrageenan polysaccharide. ${ }^{10}$ They have also studied the conformation analysis of polysaccharides and shown that most of the agarose chains make double helixes in the solid and gel state. ${ }^{4}$

Assuming that the $T_{2}$ transition in the agarose system which accompanied the optical rotation change was a coil-helix transition, as proposed by Rees in carrageenan, various data obtained in the present paper could be well interpreted: the helix formation from a random coil increases the relaxation time of the segment or the probe adsorbed on it to decrease $1 / p$ of the whole system below $T_{2}$, and brings about a change in the hydrodynamic volume of the 
Studies of Agarose Gel by Fluorescence Method. I.

agarose chain to alter the reduced viscosity. The exothermic peak at $\mathrm{T}_{2}$ in DSC curve is also reasonably interpreted as the helix formation accompanying hydrogen bonding, as suggested in the result of potassium thiocyanate solution in Figure 5. Rees, et al.," stated negative opinions about hydrogen bonding during the agarose double helix formation from the conformation analysis, but it seems more reasonable from our data to assert that hydrogen bonding takes part in the helix formation of a polysaccharide such as agarose.

In the DSC thermogram it is seen, though qualitatively, that the enthalpy of melting is much bigger than that of helix formation, and that there is no detectable enthalpy at the gel point. This suggests that another exothermic, slow gelling reaction at $T_{1}$ follows the helix formation at $T_{2}$, and hence the enthalpy of melting consists of the sum of that of helix formation and of gelation.

The fluorescence polarization method in the present paper thus provides new information about the agarose gelling system; the coil-helix transition point at $T_{2}$ is an especially interesting and important finding. These results still contain many problems to be solved: for example, the interpretation of the gelling mechanism and of the hysteresis phenomena. More detailed work is now proceeding in our laboratory and will be reported soon. It is, moreover, expected that the validity of the method will be extended to the investigation of other gelling biopolymer systems including their phase transitions.

The authors wish to express their thanks to Professor Yasunori Nishijima of Kyoto Uni- versity for his continued advice and helpful discussions. This work was supported in part by a Scientific Research Grant-in-aid of the Ministry of Education, to which the authors' thanks are due.

\section{REFERENCES}

1. (a) Y. Nishijima, Kobunshi (High Polymers, Japan), 13, 81, 166, 238 (1964); (b) Y. Nishijima, J. Macromol. Sci. Phys., B8 (3-4), 389 (1973); (c) Y. Nishijima, "Progress in Polymer Science, Japan," Vol. 6, S. Onogi and K. Uno, Eds., Kodansha, Tokyo, John Wiley, New York, N.Y., 1973, pp 199-251; (d) U. Kanaoka, et al., Eds., "Principles of Fluorescence Method and the Application to Biological Systems," Supplemental Volume of Protein, Nucleic Acid, and Enzyme, Kyoritsu Shuppan, Tokyo, 1974.

2. C. Araki and K. Arai, Bull. Chem. Soc. Jpn., 40, 1452 (1967).

3. D. A. Rees, Advan. Carbohyd. Chem., 24, 267 (1967).

4. S. Arnott, A. Fulmer, W. E. Scott, I. C. M. Dea, R. Moorhouse, and D. A. Rees, J. Mol. Biol., 90, 269 (1974).

5. S. Arnott, W. E. Scott, D. A. Rees, and C. G. McNab, ibid., 90, 253 (1974).

6. M. Duckworth and W. Yaphe, Carbohyd. Res., 16, 189 (1971).

7. N. Hirai, Nippon Kagaku Zasshi (J. Chem. Soc. Jpn., Pure Chem. Sect.) 72, 837 (1951).

8. E. Pines and W. Prins, Macromolecules, 6, 888 (1973).

9. D. A. Rees, I. W. Steele, and F. E. Williamson, J. Polym., Sci., Part C, 7 (28), 261 (1969).

10. D. A. Rees, W. E. Scott, and F. B. Williamson, Nature, 227, 390 (1971). 